\title{
Galerkin Finite Element Scheme with Bayliss-Gunzberger-Turkel-like Boundary Conditions for Vectorial Optical Mode Solver
}

\author{
H.P. Uranus ${ }^{1,2, *}$, H.J.W.M. Hoekstra ${ }^{1}$, E. van Groesen ${ }^{2}$ \\ ${ }^{1}$ Integrated Optical Micro System Group and ${ }^{2}$ Applied Analysis and Mathematical Physics Group, \\ MESA + Research Institute, University of Twente, P.O. Box 217, 7500 AE, Enschede, The Netherlands. \\ e-mail: h.p.uranus@el.utwente.nl
}

\begin{abstract}
A Galerkin finite element scheme furnished with $1^{\text {st }}$-order Bayliss-Gunzberger-Turkel-like boundary conditions is formulated to compute both the guided and leaky modes of anisotropic channel waveguides of non-magnetic material with diagonal permitivity tensor. The scheme is formulated using nodal-based transverse components of magnetic fields for quadratic triangular elements. The symmetry and shape of the structure, together with the boundary conditions have been exploited to reduce the size of the computational domain. Results for some structures will be presented. The effectiveness of the boundary conditions will be illustrated using a step index optical fiber with computational boundaries positioned near to the core, and leaky modes computation of an ARROW structure and a six-hole photonic crystal fiber.
\end{abstract}

Keywords: finite element method, boundary conditions, leaky modes, guided modes, anisotropic waveguides.

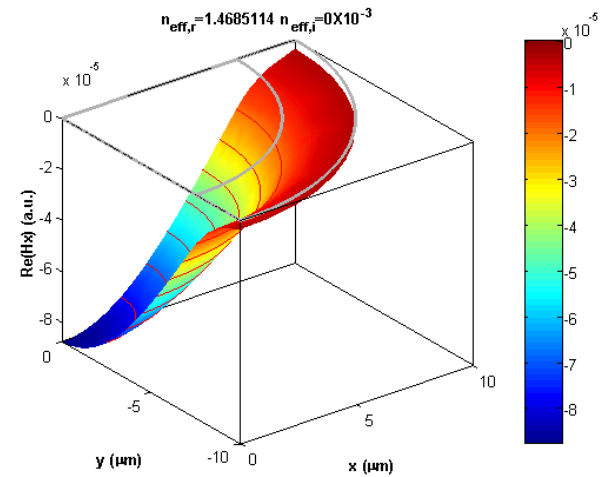

(a)

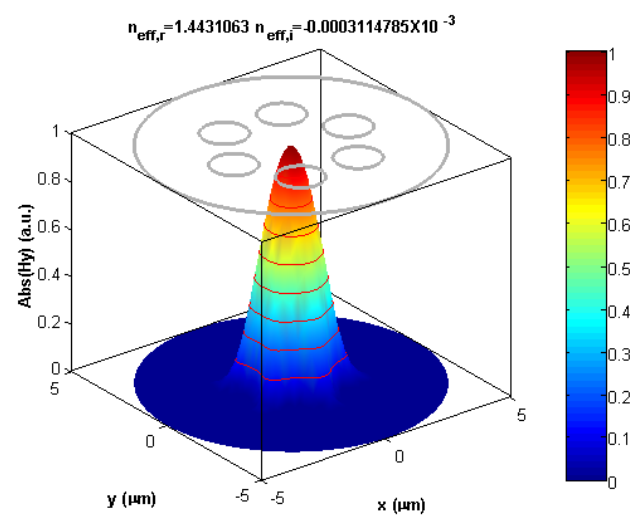

(b)

Figure 1. a. $H_{x}$ field of a step index optical fiber and b. magnitude of $H_{y}$ field of a six-hole photonic crystal fiber; both for $\mathrm{HE}_{11}$ mode
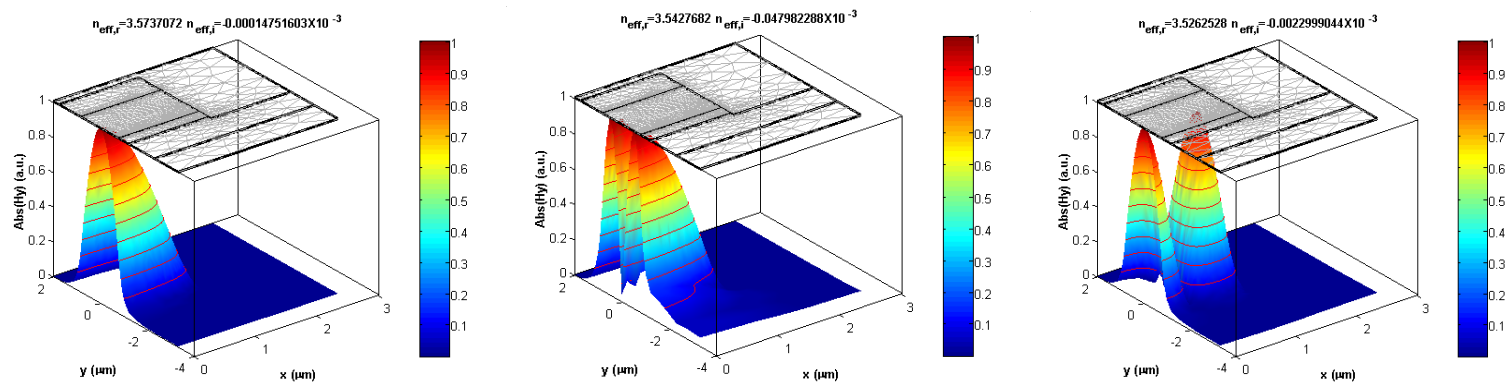

Figure 2. The magnitude of $H_{y}$ fields for leaky q-TE $\mathrm{TE}_{00}, \mathrm{q}-\mathrm{TE}_{01}$, and q-TE 20 modes of an ARROW structure. 